\title{
APPLYING OPINION MINING TO ORganize WeB OPINIONS
}

\author{
Ramandeep Sandhu ${ }^{1}$ and Rahul Mehta ${ }^{2}$ \\ ${ }^{1}$ Department of Computer Science \& Engineering, GJIET Shambhu kalan Banur, Rajpura \\ raman8deep@gmail.com \\ ${ }^{2}$ Mercer India Pvt. Ltd. Gurgaon \\ rahul.mehta@mercer.com
}

\begin{abstract}
Rapid increase of opinions on the web requires an effectual system to organize opinions. Opinion mining is a realistically plot and demanding field devoted to detect subjective content in text documents. If opinions are non-structured then it's difficult for customers and organizations to understand. This study proposes an approach focusing on designing a system to organize web opinions at the time when user is posting, before actually being extracted by expertise. New system (Opinion Organization System) provides four stages. In first stage, it provides a list of several product categories and user selects at least one. In second stage, a list of selected product relevant features is displayed to the user. In third stage, user firstly selects features for which wants to express opinions, then uses polarity based P set and $N$ set containing adjective words list and in fourth stage, uses thumb selection table to add opinions.
\end{abstract}

\section{KEYWORDS}

Opinion Mining, Opinion Organization, Opinion Extraction, Topic-Related Opinions.

\section{INTRODUCTION}

In Today's world, by the growth of popularity in opinion resources such as online news, forums, blogs and reviews, user actively seek opinions for their interest. Opinions are subjective statements that reflect people's sentiments or perceptions towards something. It is an assessment, judgment or evaluation of something. Opinions can now be found almost everywhere -blogs, social networking sites like Face book and Twitter, news portals, e-commerce sites, etc. Ecommerce facility because of World Wide Web has enhanced users and organizations satisfaction in the sense, customers are buying and organizations are selling more and more products on the web. Web providing internet is much more comfortable because when a customer wants to purchase new product then s/he doesn't has to go to shopkeeper and to friends to ask about its features and qualities. It's possible with web that by reading other's posted opinions, s/he can take decision whether to purchase new product or not. So, opinion mining information system helps potential customer to take self-decision that's why it is decision-making system. On the web, hundreds of people opinions are available. Opinion mining [1, 2] (sentiment mining, opinion/sentiment extraction) is the part of research that attempts to construct automatic systems to determine individual attitude from text written in natural language [2]. It actually identifies the user's viewpoints about a subject, rather than simply identifying the subject. It's not based on the proposal of mining keywords obtainable on the web but mining the context of available keywords. We need a well planned and controlled opinion mining system which enhance opinion mining and make it more users friendly and efficient.

DOI : 10.5121/ijcsea.2011.1408 
Various phases of opinion mining system are: opinion extraction, analysis, classification, scaling and summarization. Opinion extraction as first phase of opinion mining process is performed after all opinions are loaded by user in the table provided by our study. Each new phase is dependent on previous one and output of previous phase is input to next one. Opinion extraction is first phase and rest all phases depends on it. If extraction is not relevant then opinion mining procedure will be diverted and not all features will be topic-related. Our study is concerned with feature based opinion extraction $[2,7]$. Analysis after opinion extraction is also inevitable as ways of expressing opinions of different people are different. In $[9,13,14,15]$, we consider 2 datasets of seed words named $\mathrm{P}$ set and $\mathrm{N}$ set. With the study, it's resolved that if opinion is extracted as it should be then opinion mining process can be enhanced up to $40 \%$. Opinion extraction is on web and handling of opinions on web needs proper supervision of opinions. Our study distributed work in stages and user is concerned with stages when want to write opinions. This method is constructed for efficient and intended information gathering as apposite summarization of opinions [6] helps in future. When a new customer wants to see opinions, it will be available in separate columns and not in paragraph. It provides three columns:

Column 1: "Thumbs up" opinions

Column 2: "Thumbs down" opinions

Column 3: "Supplementary" opinions

This study is further dividing column 3 into three sub columns.

Sub column 1: positive opinions

Sub column 2: negative opinions

Sub column 3: other opinions

The table is containing those positive opinions in "Thumbs up" column and negative opinions in "Thumbs down" column having feature names offered in feature list. Column 3 named "Supplementary" opinions is basically main focusing column as it is profitable to organizations. The three columns table is giving the benefit of no time consumption in firstly reading sentence based paragraphs of opinions and then recognizing which sentence in paragraph represents user's positive opinion and which sentence represents negative opinions. Our task is trying to make opinion extraction process supple by reducing the burden on opinion extraction phase. No need to do filtering of opinions posted by user. Now, our proposed system has made it possible because opinions added in sentential form by people are in three detached columns table. Column 3 is helping in the situation when user has opinions but unable to decide whether to add in "Thumbs up" or in "Thumbs down" column. Our study helps in constructing separate opinion summarization datasets according to polarity [5] and shows that efforts required for "Supplementary" opinions column are 20\% more than for "Thumbs up" and "Thumbs down" opinion columns.

The rest of the study is organized as follows: Section 2 represents a brief overview of mining tools for mining text, data, and information on web. Our approach which we call Opinion Organization System with "Thumbs" based table is described in Section 3. Section 4 endowed with advantages of proposed system. Section 5 sums up the conclusions of the paper.

\section{RELATED WORK}

Opinion mining is a correlate high esteem of text mining and its objective is to mine reviews of various products by classifying them into positive or negative meaning from the 'subjective' terms contained in the document. Automated opinion mining can provide quick search [3] and analysis [4] results to both consumers and manufacturers (Chaovalit, 2005). Works performed for classification at the document level include [16]. It's different from our work as we are interested in opinions expressed by people on each product feature in Thumb selection table rather than in single text area. Sentence level sentiment or opinion classification is studied in [17]. Our work is different from the sentence level analysis as we identify opinions on each feature. Research on 
sentence level and even document level classification methods identifies opinion words or phrases.

Web mining [10] is the attention of text mining techniques to extract valuable knowledge from various types of data on the web, including logs, cookies, links, and contents. The current research area of Web mining includes web content mining, web structure mining and web usage mining (Kosala, 2000).

In this study, Web content mining is studied as opinions to be administrated are on web. Research on related work indicates that all are providing approaches, techniques which extract opinions on web effectively and topic-related but nobody is targeting that, if we want opinion extraction best then don't entirely contemplate on opinion extraction. Also supervise its adjoining; what are other factors near to it, whose effectiveness automatically makes opinion extraction well-organized and effective one. If a system is able to manage opinions on web properly with the consequence that user has a format to add opinions then positive oriented features of a product will be already detached from negative oriented features. So, time consumption for opinion extraction will be reduced by $50 \%$.

Opinions are user generated contents which becomes an intellectually very challenging problem for researches and this study also focus on individuals opinions and its pre processing. The task of separating opinions based on polarity [5, 8, and 12] is a genre problem. It involves firstly extraction of all opinions then goes through all and then constructing directory of positive evaluations separately from negative evaluations. This proposed work is making a system for automatically handling opinions on web.

\section{THE PROPOSED SYSTEM}

As users opinions on web are central for loyalty. It's important for companies to acquire competitive advantages. Opinion mining is opinion processing which manage unstructured data coming from the web in the same modality of structured data. A large huge of web data must be administrated properly. The following diagram Fig. 1 represents the proposed system whichswitch a large huge of web data. Figure1. Opinion Organization System

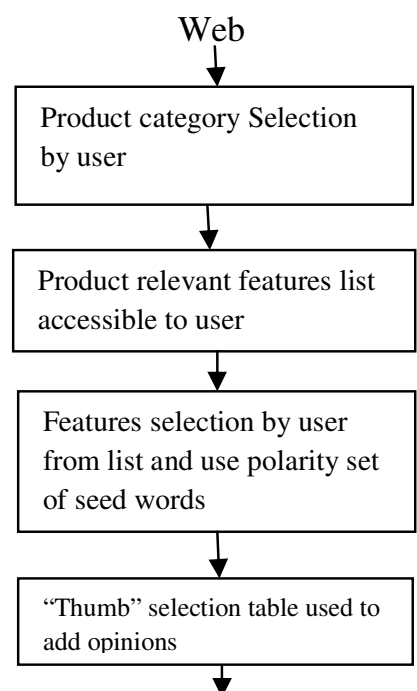

Opinion extraction and summarization process

In this section, we describe various categories of products available on web e.g. our evaluation results media, device, service, human, sports etc. Then we used feature dataset for stage 2 to provide lists of product relevant features. The product relevant features list is only visible to the 
user who wants to post opinions about a product under the selected category on the web. At single time, at least one category can be selected. This system is not helpful in the situation if user want to express opinions at a time for more than one product which comes under separate categories but helpful in the situation if products residing in same category. E.g. mobile product comes under category of media. So, user will get all feature names under media. Not all lists at same time will be visible but only after selecting a product category. Our opinion organization system is elaborated in following four steps:

On the web, user selects product category from several existing categories. E.g. user selects device category for laptop as this product is a computer device. Step 1 result all product related features for device category and it will be visible to user in a list.

User is allowed to read all features from the list but not under constraint to write for all features. User evaluates the list and selects those features for which interested to write opinions. Then uses polarity based Positive set and Negative set of seed words. User uses thumb selection table (Table 1) in which "Thumbs up", "Thumbs down" and "Supplementary" opinions columns are provided. He writes opinionated sentences by concentrating on product relevant features and different Polarity based datasets $\mathrm{P}$ set and $\mathrm{N}$ set. The Opinions added as positive perceptions under first column, negative under second and all other under third column. "Supplementary" opinions can be further of three types as positive, negative and other.

Table1. Thumb Selection Table

\begin{tabular}{|l|l|l|l|l|}
\hline $\begin{array}{l}\text { "Thumbs up" } \\
\text { opinions }\end{array}$ & $\begin{array}{l}\text { "Thumbs } \\
\text { down" } \\
\text { opinions }\end{array}$ & positive & negative & other \\
\hline & & & & \\
\hline
\end{tabular}

“Thumbs up" opinions column: Its first column of thumb selection table indicating user to write only those positive opinions whose feature name is provided in the feature list accessible to user. If product is laptop and user has five positive opinions for its separate five features but in feature list, one feature name is not existing then s/he will write only for four features and fifth feature which is not mentioned in feature list, user will not write in this column. So, "Supplementary" positive opinions sub column of third column is provided to user.

"Thumbs down" opinions column: Its second column of thumb selection table indicating user to write only those negative opinions whose feature name is provided in the feature list accessible to user. In most of the cases, negative opinions are much more than positive but repetitive because negative people have first and foremost focus on the problem that departed wrong. For laptop product, if user has three negative opinions and not any feature name is available in list then user is not allowed to use this column and all negative opinions should be written in "Supplementary" negative opinions sub column of third column as shown in table 1.

"Supplementary" opinions column: Its third column of thumb selection table helping user in following cases:

-User has positive as well as negative judgments but feature name is not available in feature list. User has option to write in positive sub column of "Supplementary" opinion column if opinionated sentence is positive and in negative sub column if opinionated sentence is negative.

-When a loyal user has opinions to write but unable to decide whether it's positive or negative. So, in such situations s/he can write in other sub column. Opinions with zero scaling (have no ranking) are neutral opinions also approaching it. 
- Set of different kind of opinions except completely positive or completely negative opinions are considered in this column. So, it solves the problem of a keyword if it is not present in domain area of particular product even then user can write opinion sentences in other sub column. It helps the organizations that at the moment of updating product relevant features in next release of feature database list, the organization will add all those features used by user and not already in existing list. It results day by day accessing people judgments and updating topic relevant features list.

The proposed system makes it possible that whatever the length of "Thumbs up", "Thumbs down" or "Supplementary" opinions (positive, negative, other) columns, these are under separate blocks. We found that during compilation process which extracts opinions column wise, "Supplementary" opinions column is focal point which requires more efforts than first and second column. Our study demonstrated that efforts requirement for "Supplementary" column is $20 \%$ high than for "Thumbs up" and "Thumbs down" column opinions because we need special system which analyzes these opinions whose chance of polarity is not completely positive and not completely negative. It is enclosure of even unconfirmed opinions in the wisdom that when user is not in agreement with polarity of opinions then he adds these in "Supplementary" column.

We give a simple example to illustrate. Assume a product laptop under device category and we provide the list of features as "price, startup, battery, hard disk, weight, size, clarity, position, zoom, resolution, quality, camera, Bluetooth, head phones, operating system and angle". We consider 2 polarity sets named $\mathrm{P}$ set and $\mathrm{N}$ set of seed words with positive and negative orientations respectively [12].

$P$ set $=\{$ long, fine, more, good, nice, excellent, positive, fortunate, correct, superior, amazing, ultimate, fascinating, charming\}

$N$ set $=\{$ short, unwell, less, bad, nasty, poor, negative, unfortunate, wrong, inferior, horrible, cruel, awful\}

In an example: one individual user has in mind many assessments for laptop product. User reads the feature list and selects features for laptop \{price, quality, battery, startup, processor, hard disk, weight and size . Then user go through $\mathrm{P}$ set and $\mathrm{N}$ set of seed words. User has following opinion sentences: It's expensive product, Superior quality laptop it is, Battery backup is high, Not latest processor, Hard disk is too much, Laptop has less weight, Size is ok, It's very durable laptop, Startup is long, Good look, Ultimate speaker, It's a working laptop, It's taking input.

As user reviews shows that no feature is available for durability, expensiveness, processor, speaker, look but user has picky kind of reviews for these features also. In such situation, our system allows user to use "Supplementary" column to add reviews. User heaps opinions in thumb selection table as represented in Table 2. 
Table2. Individual's Reviews in Thumb Selection Table

\begin{tabular}{|c|c|c|c|c|}
\hline \multirow{2}{*}{$\begin{array}{l}\text { "Thumbs up" } \\
\text { opinions }\end{array}$} & \multirow{2}{*}{$\begin{array}{l}\text { "Thumbs } \\
\text { down" } \\
\text { opinions }\end{array}$} & \multicolumn{3}{|c|}{ "Supplementary" opinions (No Thumb) } \\
\hline & & positive & negative & other \\
\hline $\begin{array}{l}\text { - Superior } \\
\text { quality laptop it } \\
\text { is. } \\
\text {-Battery backup } \\
\text { is high. } \\
\text { - Hard disk is } \\
\text { too much. } \\
\text { - Laptop has } \\
\text { more weight. }\end{array}$ & $\begin{array}{l}\text { - Startup is } \\
\text { long. }\end{array}$ & $\begin{array}{l}\text {-It's very } \\
\text { durable laptop. } \\
\text {-Ultimate } \\
\text { speaker. }\end{array}$ & $\begin{array}{l}\text {-It's expensive } \\
\text { product. } \\
\text { - Not latest } \\
\text { processor. }\end{array}$ & $\begin{array}{l}\text {-Size is ok. } \\
\text { - Good Look. } \\
\text {-It's a working } \\
\text { laptop. } \\
\text {-It's } \quad \text { taking } \\
\text { input. }\end{array}$ \\
\hline
\end{tabular}

\section{ADVANTAGES OF PROPOSED SYSTEM}

This proposed system provides following advantages:

1. It formulates the topic-relevant opinion extraction as it helps user having product opinions to assert has to firstly select category of that product. Under category, user has list of only product relevant features list.

2. Separate columns for different polarity opinions. It helps organizations to access and update regularly those features which an ordinary user is thinking but not available in feature list.

3. It avoids false positive or false negative opinions. If a user has same adjectives used in two senses like one in positive and other in negative sense then thumb selection table helps by providing separate space for positive feelings and for negative feelings. E.g. opinion (1) and (2):

The battery backup is high- (1)

The time consumption to do installation is high- (2).

It's clear that (1) is expressing positive and (2) is expressing negative opinions. Thumb selection table makes its differentiation possible.

4. Its time saving system as user has expedient features list provided in dataset. It depends on user whether to use opinion keywords from existing list or not.

5. User is not restraint to post opinions using only existing keywords. Even user is allowed to post opinions using own keywords in sentence.

6. It resolves one drawback of existing system that in a specific domain, a given adjective may have a different meaning from another domain.

7. Its efficient system for (a) Person who want to post opinions as posting positive opinions separately from negative opinions. (b) Reviewers as an organization or customer e.g. Dell company reviews posted opinions to make more efficient system with the help of those people's feelings that are using their products. New customer reviews existing opinions when going to purchase a product. (c) Expertise who does opinion summarization [6] on the web. 


\section{CONCLUSION}

The work presented here depends on the thumb selection table and other features interconnected to opinion administration on the web for getting efficient opinion mining system as a result. The system gave the less sustained and most proficient opinion organization stages with elevated ascendancy. The work is making opinion extraction more efficient by focusing on its prior era, in which by helping users having opinions about products, opinion extraction is elevated on web. Our system is providing list of product relevant features to user which allocates thumb selection table to post accurate opinions for helping others in future.

\section{ACKNOWLEDGEMENTS}

We thank Sh. Ranjit Singh Sandhu and my colleague Ms. Namita Sota for sharing ideas and helping us in literature survey. Especially, we are thankful to Gian Jyoti Group of Institutions supporting this research by providing free time in campus.

\section{REFERENCES}

[1] PANG, B. AND LEE, L. 2008. Opinion mining and sentiment analysis. Found. Trends Inf. Retr. 2, $1-2,1-135$.

[2] POPESCU, A.-M. AND ETZIONI, O. 2005. Extracting product features and opinions from reviews. In HLT '05: Proceedings of the conference on Human Language Technology and Empirical Methods in Natural Language Processing. Association for Computational Linguistics, Morristown, NJ, USA, 339-346.

[3] J Liu, G Wu, J Yao, "Opinion Searching in Multi-product Reviews", proceedings of the sixth IEEE International Conference on Computer and Information Technology, 2006.

[4] B Liu, M Hu, J Cheng, "Opinion observer: analyzing and comparing opinions on the Web", Proceedings of the 14th international conference on WWW, May 10-14, 2005.

[5] Wilson, Theresa, Janyce Wiebe, and Paul Hoffman. 2005. Recognizing contextual polarity in phrase-level sentiment analysis. In Proceedings of the Human Language Technologies Conference/Conference on Empirical Methods in Natural Language Processing (HLT/EMNLP2005), pages 347-354, Vancouver, Canada.

[6] STOYANOV, V. AND CARDIE, C. 2006b. Toward opinion summarization: linking the sources. $9-14$

[7] Popescu, "Extracting Product Features and Opinions from Reviews", Oren Etzioni, Proceedings of HLT-EMNLP, 2005.

[8] Yu, H. and Hatzivassiloglou, V. 2003. Towards Answering Opinion Question: Separating Facts from Opinions and Identifying the Polarity of Opinion Sentences, In EMNLP 2003.

[9] Hatzivassiloglou and K. McKeown. Predicting the semantic orientation of adjectives. In Proceedings of 35th Meeting of the Association for Computational Linguistics, Madrid, Spain, 1997.

[10] Raymond Kosala and Hendrik Blockeel. 2000. Web Mining Research: A Survey. SIGKDD: SIGKDD Explorations: Newsletter of the Special Interest Group (SIG) on Knowledge Discovery \& Data Mining, ACM, Vol 2, No 1, pp. 1-15.

[11] Gamon, M.; Aue, A.; Corston-Oliver, S.; and Ringger, E. K. 2005. Pulse: Mining customer opinions from free text. In Proceedings of the 2005 Conference on Intelligent Data Analysis (IDA), 121.132.

[12] P. Turney. Thumbs up or thumbs down? Semantic orientation applied to unsupervised classification of reviews. In Proceedings of 40th Meeting of the Association for Computational Linguistics, pages 417-424, Paris, 2002 
International Journal of Computer Science, Engineering and Applications (IJCSEA) Vol.1, No.4, August 2011

[13] J. Kamps, M. Marx, R. J. Mokken, and M. Rijke. Using wordnet to measure semantic orientation of adjectives. In Proceedings of LREC 2004, the 4th International Conference on Language Resources and Evaluation, pages 174-181, Lisbon, Portugal, 2004.

[14] M. Taboada, C. Anthony, and K. Voll. Creating semantic orientation dictionaries. 2006.

[15] K. Voll and M. Taboada. Not all words are created equal: Extracting semantic orientation as a function of adjective relevance. Pages 337-346. Volume 4830/2007 AI 2007: Advances in Artificial Intelligence, 2007.

[16] A-M. Popescu and O. Etzioni. Extracting Product Features and Opinions from Reviews. EMNLP$05,2005$.

[17] S. Morinaga, K. Yamanishi, K. Tateishi, and T. Fukushima, Mining Product Reputations on the Web. KDD’02, 2002

\section{Authors}

Ms. Ramandeep Sandhu is a Lecture in Computer Science Department at Gian Jyoti Institute of Engg \& Tech, Shambhu Kalan Banur (Rajpura). She is pursuing her M. Tech from GNE College, Ludhiana with agg. $79 \%$ with $1^{\text {st }}$ rank in class. Her research interest is Data Mining, Opinion Mining and Software complexity measures. She has published 01 paper in International Journal IJCSEA, published 01 International Paper, presented and published 05 National papers and presented 01 National Seminar on Opinion Mining in Punjab. You can contact her at Contact her at raman8deep@gmail.com

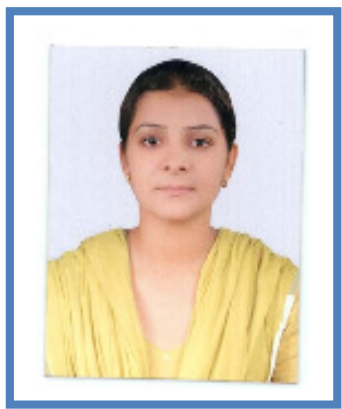

